\title{
Inconsistent Global Kinetic Energy Spectra in Reanalyses and Models
}

\author{
JiH-WANG AARON WANG ${ }^{\mathrm{a}, \mathrm{b}}$ AND PRASHANT D. SARDESHMUKH ${ }^{\mathrm{a}, \mathrm{b}}$

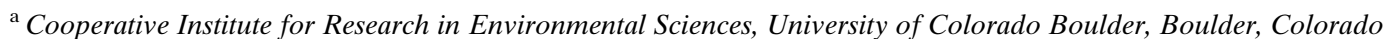 \\ ${ }^{\mathrm{b}}$ NOAA/Earth System Research Laboratories/Physical Sciences Laboratory, Boulder, Colorado
}

(Manuscript received 2 October 2020, in final form 14 May 2021)

\begin{abstract}
Global upper-tropospheric kinetic energy (KE) spectra in several global atmospheric circulation datasets are examined. The datasets considered include ERA-Interim, JRA-55, and ERA5 and two versions of NOAA GFS analyses at horizontal resolutions ranging from $0.7^{\circ}$ to $0.12^{\circ}$. The mesoscale portions of the spectra are found to be highly inconsistent. This is shown to be mainly due to inconsistencies in the scale-dependent numerical damping and in the large contributions to the global mesoscale KE from the KE in convective regions and near orography. The spectra also generally have a steeper mesoscale slope than the $-5 / 3$ slope of the observational Nastrom-Gage spectrum pursued at many modeling centers. The sensitivity of the slope in global models to 1) stochastically perturbing diabatic tendencies and 2) decreasing the horizontal hyperviscosity coefficient is explored in large ensembles of 10-day forecasts made with the NCEP GFS $\left(0.7^{\circ}\right.$ grid $)$ model. Both changes lead to larger mesoscale KE and a flatter spectral slope. The effect is stronger in the modified hyperviscosity experiment. These results show that (i) despite assimilating vastly more observations than used in the original NastromGage studies, current high-resolution global analyses still do not converge to a single "true" global mesoscale KE spectrum, and (ii) model KE spectra can be made flatter not just by increasing model resolution but also by perturbing model physics and decreasing horizontal diffusion. Such sensitivities and lack of consensus on the spectral slope also raise the possibility that the true global mesoscale spectral slope may not be a precisely $-5 / 3$ slope.
\end{abstract}

KEYWORDS: Convection; Diffusion; Kinetic energy; Spectral analysis/models/distribution; Reanalysis data; Stochastic models

\section{Introduction}

Accurate representation of the global atmospheric kinetic energy (KE) wavenumber spectrum is considered essential in weather and climate models, partly because it establishes saturation bounds for forecast errors at each scale (e.g., Lorenz 1969; Lilly 1972). Errors in model spectra are often interpreted as resulting from misrepresentations of multiscale interactions. Such interactions are associated not only with turbulent energy cascades across scales but also with diabatic processes and interactions with orography (Zhang et al. 2003, 2007; Judt 2018). Most previous discussion on this topic has focused on the nature and representation of the cascades, in particular the upscale energy and downscale enstrophy cascades in inertial wavenumber ranges anticipated by large-scale two-dimensional turbulence theory. Relatively little attention has been given to the contributions to the global spectrum by diabatic processes and interactions with orography. We find here that these contributions are both important and highly uncertain, not only in models but also in emerging high-resolution global analysis datasets. There is consequently a disturbing lack of consensus among the analyses as to whether the true mesoscale spectral slope is the $-5 / 3$ slope (on a log-KE vs log-wavenumber plot) pursued at modeling centers (e.g., Dueben et al. 2020; Skamarock et al. 2014; Ji et al. 2016; Kitamura and Matsuda 2010).

Given the approximately two-dimensional character of atmospheric flow, much of our intuition about what the atmospheric KE spectrum should look like comes from the theory of

Corresponding author: Jih-Wang Aaron Wang, aaron.wang@ noaa.gov isotropic and homogeneous two-dimensional turbulence (e.g., Kraichnan 1967). Kraichnan predicted $-5 / 3$ and -3 spectral slopes in postulated inertial ranges of constant spectral energy and enstrophy fluxes at low and high wavenumbers, respectively. Such slopes are indeed evident in upper-tropospheric observations (e.g., Nastrom et al. 1984; Nastrom and Gage 1985; Lindborg 1999), but in the "wrong" wavenumber ranges: the steep -3 slope is most evident at lower subsynoptic wavenumbers, whereas the flatter $-5 / 3$ slope is most evident at higher mesoscale wavenumbers. Many attempts have been made to reconcile this discrepancy, including questioning the theoretical assumption of inertial ranges of constant spectral fluxes, for which there is no compelling observational evidence. Indeed, some studies (e.g., O'Gorman and Schneider 2007) have questioned whether spectral fluxes (let alone constant spectral fluxes) are relevant at all in determining, e.g., the -3 slope of the observed subsynoptic spectrum.

On the other hand, it is also not clear to what extent the accuracy of previously published observational spectra is affected by data gathering techniques and limited space and time sampling. Nastrom and Gage (1985) Fourier analyzed wind observations along commercial flight routes and estimated the global spectral slope in the upper troposphere and lower stratosphere to be about $-5 / 3$ at scales smaller than $300-$ $400 \mathrm{~km}$ and about -3 at larger scales. Lindborg (1999) obtained generally consistent results using independent global observations (with some concentration over North America, Europe, and the northern Atlantic Ocean), as did Cho et al. (1999a,b) with observations over the Pacific Ocean. Frehlich and Sharman (2010) performed an extensive study of rawinsonde and Aircraft Communications, Addressing, and Reporting 
System/Aircraft Meteorological Data Relay (ACARS/AMDAR) observations in terms of horizontal structure functions (that are variances of two-point wind differences plotted as a function of the separation distance between the points), whose slopes are related to the slopes of the KE spectra. They found the slopes to be generally consistent with previous studies, albeit with some dependence on latitude, season, topography, and altitude for separation distances between 20 and $400 \mathrm{~km}$. Nevertheless, the aircraft observations used in all these studies had sampling deficiencies in time and space, as acknowledged by the authors, and the spectra estimated by Fourier analyzing observations taken along flight routes and then averaged may not have been representative of the global or even the respective regional $\mathrm{KE}$ spectra.

Some aspects of the theoretical and observed KE spectra can be reproduced in simple numerical quasigeostrophic models [e.g., Tung and Orlando 2003; see also Smith (2004) and Tung (2004) for a discussion of the spectrum sensitivity to numerical dissipation]. This approximate consistency among observations, simple theory, and simple numerical models has encouraged the pursuit of even greater consistency between observations and comprehensive general circulation model (GCM) simulations (e.g., Lauritzen et al. 2011; Thatcher and Jablonowski 2016; Skamarock et al. 2014). In particular, it is presumed that the simulated KE spectral slope will approach -3 at subsynoptic and $-5 / 3$ at mesoscale wavenumbers as model resolution is increased. This assumes of course that the adiabatic dynamical cascades are dominant at these wavenumbers, and that diabatic processes, frictional dissipation, and orographic interactions are less important.

In recent decades, the increased resolution of GCMs has enabled major weather centers around the world to produce highresolution, high-quality analyses of global upper-tropospheric wind fields. The analyses generally assimilate satellite-observed atmospheric motion vectors (AMVs), ACARS/AMDAR data, other conventional upper-air wind observations (from, e.g., wind profilers and rawinsondes), plus numerous observations of other meteorological variables that are physically related to the winds, and combine them with model-generated guess fields (typically 6-h forecasts from a comprehensive numerical weather prediction model) to create a final dynamically consistent product that accounts for inevitable errors in both the observations and the guess fields. Such analyses are increasingly useful for estimating the subsynoptic/mesoscale KE spectrum, since they are less impacted at these scales by the models' admittedly ad hoc scale-dependent numerical dissipation used to control spurious buildup of energy at the highest resolved wavenumbers. One might therefore expect them to represent the cascades better than the previous generation of lowerresolution reanalyses, as well as the diabatic, frictional, and orographic contributions to the spectrum at the lower and better-resolved subsynoptic/mesoscale wavenumbers.

Despite this expectation, we find here that the mesoscale portions of the spectra are highly inconsistent among such analyses, even though their resolution extends well into mesoscales. To avoid confusion in the discussion to follow, we define mesoscales as corresponding to total spherical wavenumbers $n$ between 101 and 2000 (wavelengths $L$ between 400 and $20 \mathrm{~km})$, subsynoptic scales as wavenumbers $64-100(L \sim$ $625-400 \mathrm{~km})$, synoptic scales as wavenumbers $20-63(L \sim$ $2000-625 \mathrm{~km}$ ), and large scales as wavenumbers lower than 20 $(L>2000 \mathrm{~km})$. We explore the sources of the spectral inconsistencies among the analyses, and also some sensitivities of the mesoscale spectrum to model formulation.

Section 2 compares the 200-hPa KE spectra in the analyses and shows how their inconsistent global mesoscale KE and spectral slopes are associated with inconsistent regional mesoscale KE and spectral slopes in regions of deep convection and near orography. Section 3 further highlights the sensitivity of the mesoscale spectral slopes to the complex physical processes in such regions by comparing large ensembles of forecasts made with and without additional stochastic perturbations to the parameterized physical tendencies in a quasi-operational global weather model. Section 4 shows the sensitivity of the mesoscale KE and spectral slopes in the same model to changes in the model's horizontal hyperdiffusion coefficient. Given such model sensitivities and the large and inconsistent diabatic contributions to the global mesoscale KE from deep convective regions in even relatively high-resolution analyses and models, we suggest in section 5 that a $-5 / 3$ global mesoscale spectral slope pursued as "truth" at modeling centers may perhaps not be a good discriminating metric of model realism.

\section{Mesoscale KE differences among reanalysis datasets}

We first describe the common period of study for which all KE spectra were computed using various analysis datasets and model forecasts. This is followed by analysis intercomparison and interpretation of the large mesoscale KE differences, especially in convective regions and near orography. The credibility and the effective resolutions of the analyses are discussed in sections $2 \mathrm{c}$ and $2 \mathrm{e}$.

\section{a. Period of study}

All KE spectra presented here were computed using 12-hourly global $200-\mathrm{hPa}$ wind fields in the 4 February to 14 March 2016 period. In this period the El Niño Rapid Response (ENRR) field campaign (Dole et al. 2018) over the eastern Pacific provided additional observations, which were assimilated along with the abundant routine global observations to generate global wind analyses using the Global Forecast System (GFS) model and data-assimilation system developed at the National Centers for Environmental Prediction (NCEP) of the National Oceanic and Atmospheric Administration (NOAA). We used two types of GFS analyses for this period: a low-resolution ("low-res") analysis and a high-resolution ("high-res") analysis. Both types were described and investigated extensively in recent studies (Slivinski et al. 2019; Wang et al. 2019).

\section{b. Analysis datasets used}

Table 1 summarizes the resolutions and output grids of the global analyses used in this study. More details are provided below, mainly for the convenience of those who may wish to reproduce our calculations. Others may skip to section 2c. 
TABLE 1. List of analysis and reanalysis products utilized; $J$ is the number of grid points in the meridional direction.

\begin{tabular}{lccccc}
\hline \hline \multicolumn{1}{c}{ Analysis } & $\begin{array}{c}\text { Spectral model } \\
\text { resolution }\end{array}$ & $\begin{array}{c}\text { Analysis increment } \\
\text { grid/resolution }\end{array}$ & Original output grid & Horizontal interpolation & Final output grid \\
\hline Low-res GFS & T254 & F128; $J=256$ & F128; $J=256$ & Bilinear & $0.5^{\circ} \times 0.5^{\circ} ; J=361$ \\
High-res GFS & T1534 & F288; $J=576$ & F768; $J=1536$ & Bilinear & $0.25^{\circ} \times 0.25^{\circ} ; J=721$ \\
ERAI & T255 & T95 and T159 & F128; $J=256$ & None & F128; $J=256$ \\
ERA5 & T639 & T95, T159, and T255 & F320; $J=640$ & None & F320; $J=640$ \\
JRA-55 & T319 & T106 & N160; $J=320$ & Cubic along latitude circles & F160; $J=320$ \\
\hline
\end{tabular}

\section{1) GFS ANALYSES}

The low-res GFS analysis was generated using the T254 spectral GFS model $\left(\sim 0.71^{\circ}\right.$ grid) for the atmospheric dynamics, and an N128 reduced Gaussian grid (with 256 meridional grid points) for the nonlinear dynamical terms, physical parameterizations, and boundary conditions. The data-assimilation method was a hybrid four-dimensional ensemble variational (4D-EnVar) method (Buehner et al. 2013; Kleist and Ide 2015), based on using an 80-member ensemble of short-term global forecasts to generate an 80 -member ensemble of global atmospheric analyses every $6 \mathrm{~h}$. The analysis was performed on an F128 regular Gaussian grid (256 meridional grid points). The wind analysis was then bilinearly interpolated to a fixed $0.5^{\circ}$ longitude $\times 0.5^{\circ}$ latitude grid (hereafter $0.5^{\circ} \times 0.5^{\circ}$ grid) We note that bilinear interpolation can have some effect on the estimated KE spectrum at the highest wavenumbers, but not enough to alter our principal conclusions.

The high-res GFS analysis shared the same dynamical core and similar physics as the low-res analysis, but used a higherresolution model and a slightly different data-assimilation method. Specifically, it used a T1534 spectral discretization $\left(\sim 0.12^{\circ}\right.$ grid $)$ for the atmospheric dynamics and an N768 reduced Gaussian grid (1536 meridional grid points) for the nonlinear dynamical terms, physical parameterizations, and boundary conditions. Like the low-res analysis, it also used a 4D-EnVar data-assimilation method; however, the ensemble background-error covariances were weighted averages of climatological background-error covariances and covariances derived from the ensemble spread of 80 -member ensembles of lower-resolution T574 $\left(\sim 0.31^{\circ}\right)$ short-term forecasts. The analysis was performed on an F288 regular Gaussian grid (576 meridional grid points). Unlike the lowres analysis, an incremental analysis update (IAU; Bloom et al. 1996; Lei and Whitaker 2016; Takacs et al. 2018) was not used in the high-res analysis; instead, a digital filter was applied to the background forecast fields to prevent highfrequency noise (Lynch and Huang 1992; Huang and Lynch 1993). The 4D-EnVar analysis was performed using only the control forecast at T1534 resolution, hence only one highres analysis was generated in every analysis cycle. The wind analysis output was first projected to an F768 regular Gaussian grid and then bilinearly interpolated to a fixed $0.25^{\circ}$ longitude $\times 0.25^{\circ}$ latitude grid (hereafter $0.25^{\circ} \times 0.25^{\circ}$ grid). We also note that because of the horizontal hyperdiffusion employed, the high-res GFS compared to the lowres GFS damps the highest resolved wavenumbers relatively strongly and lower wavenumbers relatively weakly. This can have a differential impact on the KE spectrum in the analyses, which we discuss later in sections $2 \mathrm{c}$ and 4 .

\section{2) ECMWF REANALYSES}

The European Centre for Medium-Range Weather Forecasts (ECMWF) interim reanalysis (hereafter ERAI; Dee et al. 2011), and its successor fifth generation of ECMWF atmospheric reanalysis (hereafter ERA5; Hersbach et al. 2018, 2020), were both used to compute KE spectra. The ERAI system used a T255 spectral model ( $\sim 0.7^{\circ}$ grid) for the atmospheric dynamics and an N128 reduced Gaussian grid for the nonlinear dynamical terms, physical parameterizations, and boundary conditions. The analysis increment was obtained by running two successive inner loops at resolutions of T95 and T159 to minimize the cost function. The ERA5 system used a T639 spectral model $\left(\sim 0.28^{\circ}\right.$ grid) and an N320 reduced Gaussian grid, and the analysis increment was obtained by running three successive inner loops at resolutions of T95, T159, and T255 to minimize the cost function. The consistency of ERAI and ERA5 in computational work flow, spectral method, use of a reduced Gaussian grid, etc., facilitate a relatively clean assessment of the impact of resolution on KE spectra. More details of these reanalyses can be found on the ECMWF website (see https://confluence.ecmwf.int/pages/ viewpage.action?page $I d=74764925)$.

For this study, the ERAI wind dataset was downloaded directly from the ECMWF website (https://apps.ecmwf.int/ datasets/data/interim-full-daily/levtype $=\mathrm{pl} /$ ), whereas the ERA5 wind dataset was downloaded from the Climate Data Store at the ECMWF website (https://cds.climate.copernicus.eu/ cdsapp\#!/dataset/reanalysis-era5-single-levels?tab=form). The web API service provides an option to download the data on Gaussian or fixed (e.g., regular latitude-longitude) grids. We note that our downloaded data on Gaussian grids correspond to their full (untruncated) spectral resolutions, whereas similar data from other data repositories may be truncated at some wavenumber [e.g., ERAI from University Corporation for Atmospheric Research (UCAR) truncated at $n=170$ ] to eliminate nonlinear aliasing (D. Stepaniak, UCAR, 2018, personal communication), or may only be available on fixed grids (ERA5 from UCAR on a $0.25^{\circ}$ latitude-longitude grid).

\section{3) JMA REANALYSIS}

Similar to the ECMWF reanalyses, the Japanese Meteorological Agency (JMA) 55-year Reanalysis (JRA-55) also used a spectral model for the atmospheric dynamics and a reduced Gaussian grid for the nonlinear dynamical terms, physical parameterizations, and boundary conditions. The model guess 
fields were generated with a T319 spectral $\left(\sim 0.56^{\circ}\right.$ grid $)$ model, and a $4 \mathrm{D}-$ Var data-assimilation method was used to produce the analysis increments (Kobayashi et al. 2015). The analysis increments were computed at T106 resolution. The original JRA-55 output on an N160 reduced Gaussian grid was interpolated to an F160 regular Gaussian grid by cubic interpolation along latitude circles, approximately equivalent to Fourier interpolation (D. Stepaniak 2020, personal communication). The JRA-55 wind fields on the regular Gaussian grid on model levels were downloaded from the UCAR website (https:// rda.ucar.edu/datasets/ds628.0/), and were vertically interpolated to the $200-\mathrm{hPa}$ pressure level using logarithmic interpolation.

\section{c. Spectra}

\section{1) KE SPECTRA}

To compute the KE spectra, the 200-hPa wind fields in all datasets were first converted to vorticity and divergence using spherical harmonics. The squared magnitudes of their spherical harmonic coefficients were then summed over $m$ and divided by $n(n+1) / a^{2}$, where $m$ is zonal wavenumber, $n$ is total wavenumber, and $a$ is Earth's radius, to obtain the KE of the rotational and divergent flow, respectively, at each $n$. Their sum represents the KE of the full horizontal flow at each $n$.

Although $n$ can theoretically range from 0 to infinity, in practice $n$ ranges from 0 to the number of latitudes on the final output grid of each analysis dataset. For example, the high-res GFS analysis winds were interpolated from the F768 regular Gaussian grid (1536 latitudes) to the $0.25^{\circ} \times 0.25^{\circ}$ grid $(721$ latitudes including the poles); hence $n$ ranged from 0 to 721 in that case. Table 1 shows the resolutions of the $200-\mathrm{hPa}$ analysis wind fields and also of the analysis increments (i.e., the analysis minus guess fields), together with their final output grids.

Figure 1a shows the average global 200-hPa KE spectrum in each of our analysis datasets (low-res GFS, high-res GFS, ERAI, ERA5, and JRA-55), determined as the average of the 80 twelve-hourly spectra in the 4 February to 14 March 2016 period. Note the logarithmic axes. There is general agreement on large scales $(n<20)$, minor but noticeable disagreement on subsynoptic scales $(64 \leq n \leq 100)$, and very large disagreement on mesoscales $(n>100)$. For example, the ratio of the ERA5 energy (green thick solid line) to the ERAI energy (blue thick solid line) exceeds $10^{0.63}=4.25$ at $n=100$ and $10^{1.68}=47$ at $n=170$.

Given such large inconsistencies among the mesoscale spectra in Fig. 1a, it is hard to escape the conclusion that the true global mesoscale KE spectrum remains basically unknown at present. The figure also suggests, contrary to common belief, that higher model resolution alone does not guarantee larger subsynoptic KE magnitude. For example, the high-res GFS (T1534) and JRA-55 (T319) analyses have lower subsynoptic KE than the low-res GFS (T254) analysis.

Figure 1a also includes a KE spectrum derived from day-10 high-res (T1534) GFS forecasts generated using the high-res GFS analyses as initial conditions. It is slightly higher than the high-res GFS analysis spectrum, but is still lower than the T639 ERA5 spectrum at mesoscales and still has a steeper than $-5 / 3$

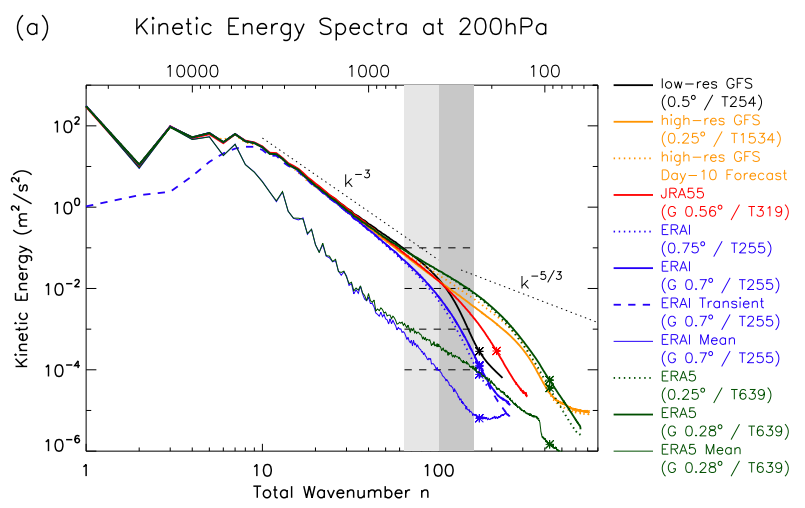

(b) Potential Temperature Spectra at $200 \mathrm{hPa}$

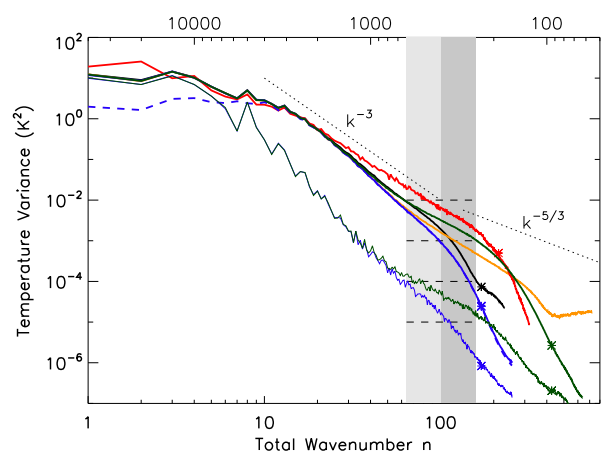

FIG. 1. (a) Mean global 200-hPa KE spectra determined from 12-hourly low-res and high-res GFS analyses, day-10 high-res GFS forecasts, and JRA-55, ERAI, and ERA5 in the 4 Feb to 16 Mar 2016 period. The two lowest curves show the KE spectrum of the time-mean 200-hPa winds during the period in the ERAI and ERA5. The dashed blue curve shows the KE of the transient 200-hPa winds in the ERAI, defined as departures from the timemean winds during the period. Straight dotted line segments illustrate $n^{-3}$ and $n^{-5 / 3}$ spectral slopes. Asterisk symbols beyond $n=100$ indicate the effective nonaliasing spectral resolutions of the low-res GFS and ERAI $(n=170)$, JRA-55 $(n=213)$, and ERA5 $(n=426)$. The two vertical gray-shaded bars demarcate the subsynoptic-scale wavenumber band $1(64 \leq n \leq 100)$ and mesoscale wavenumber band $2(101 \leq n \leq 158)$ investigated in greater detail in the rest of the paper. Numbers in parentheses on the right indicate "final" output grid sizes after interpolation and transformation to regular or Gaussian ("G") grids, and the numbers to their right the nominal spectral resolutions of the datasets. For reasons given in the text, the ERAI spectrum determined from the $0.75^{\circ} \times 0.75^{\circ}$ output grid is cut off at $n=200$, and the low-res GFS spectrum determined from the $0.5^{\circ} \times 0.5^{\circ}$ output grid is cut off at $n=230$. (b) As in (a), but for 200-hPa potential temperature spectra.

slope at $n=170$. Note that although there is no predictability of mesoscale motions at day 10 , this spectrum should not be interpreted as the spectrum of a free-running high-resolution model, because it is affected at larger scales by observed initial conditions and at smaller scales by the downscale cascades over 10 days from those larger scales.

It is important to recognize that the mesoscale portions of the analysis spectra in Fig. 1a are influenced to varying degrees by the effective resolution of the analyses being lower than their nominal resolutions of T255 (ERAI), T254 (low-res 
GFS), T319 (JRA-55), T639 (ERA5), and T1534 (high-res GFS). This effective resolution can be hard to quantify, because there is more than one way to define it. One way is to identify it with the highest nonaliased wavenumber. For example, wavenumber $n=170$ is the highest nonaliased wavenumber in the ERAI and low-res GFS analysis according to the following formula for linear grids (e.g., Gaussian quadrature grids and regular latitude-longitude grids):

$$
n_{\text {eff }}=\frac{2 n_{\text {lat }}-1}{3} \approx 170
$$

where $n_{\text {lat }}$ is the number of grid latitudes between the poles (D. Stepaniak 2018, personal communication; see also Randall 2017). As such, $n=170$ may be interpreted as the highest credible wavenumber in the ERAI and low-res GFS analyses, and thus their effective resolution as being T170, not T255 or T254. Using the same criterion, the effective resolutions of JRA5 and ERA5 are then higher than T170, but lower than their nominal resolutions of T319 and T639, respectively. A second way to define the effective analysis resolution is to identify it with the resolution of the analysis increments, since it is only on these scales that the analyses are directly affected by the observations in each data-assimilation cycle. As indicated in Table 1, the analysis increments in our datasets are generally produced at a lower resolution than the background forecast guess fields (except the low-res GFS analysis). This too results in the effective analysis resolution being lower than the nominal resolution, although precisely by what amount is difficult to quantify, because of the downscale impacts of the largerscale analysis increments in succeeding assimilation cycles.

With these considerations in mind, we conservatively estimate the effective resolution of all the analyses considered here to be at least T170. (We postpone discussion of another even more conservative but arguably less justifiable way to define the effective resolution until section 2e.) The large inconsistencies in Fig. 1a beyond wavenumber $n=170$ are mostly due to model inconsistencies which, although interesting, are not our principal focus here. Rather, our focus is on the large inconsistencies at subsynoptic and mesoscale wavenumbers, specifically in the $64 \leq n \leq 100$ and $101 \leq n \leq 158$ wavenumber ranges indicated by the gray vertical shaded bars in the figure.

\section{2) Potential temperature (THETA) SPECTRA}

For completeness, Fig. 1b also shows the average global 200-hPa potential temperature (theta) spectrum in each of our datasets in a similar format to that of Fig. 1a. Similar to the KE spectra, the theta spectra also show general agreement on large scales $(n<20)$, minor but noticeable disagreement on subsynoptic scales $(64 \leq n \leq 100)$, and very large disagreement on mesoscales $(n>100)$. For a reason unclear to us, the JRA-55 spectrum is an outlier in this figure, with higher values and a nearly constant -3 slope in the $20<n<200$ wavenumber range. Similar to the discussion of the KE spectra, the large inconsistencies in Fig. 1b suggest that the true global mesoscale theta spectrum remains basically unknown at present. The figure similarly suggests that higher resolution alone does not

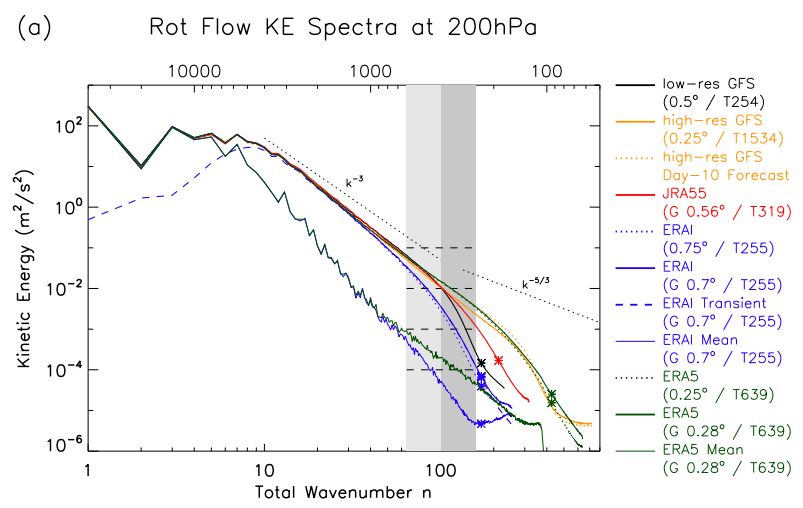

(b) Div Flow KE Spectra at 200hPa

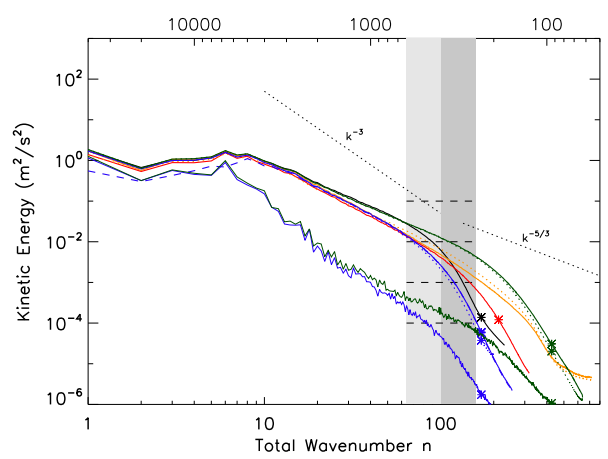

FIG. 2. As in Fig. 1a, but for the 200-hPa (a) rotational and (b) divergent flow.

guarantee larger subsynoptic theta variance. For example, the high-res GFS (T1534) analysis has a lower subsynoptic theta variance than JRA-55 (T319) and ERA5 (T639).

\section{3) Rotational AND DiVERGEnT KE SPECTRA}

Figure 2 shows the contributions to the global 200-hPa KE spectra in Fig. 1a from the rotational and divergent wind components. Similar to Fig. 1a, there is general agreement in both on large scales $(n<20)$, minor but noticeable disagreement on subsynoptic scales $(64 \leq n \leq 100)$, and very large disagreement on mesoscales $(n>100)$. The rotational flow KE dominates at large $(n<20)$ and synoptic $(20 \leq n \leq 63)$ scales, but is comparable to the divergent flow $\mathrm{KE}$ at smaller scales $(n>64)$. Consequently, the divergent flow spectrum has a shallower slope than the rotational flow spectrum in the $20<$ $n<200$ wavenumber range in all the analyses. Indeed, the slope of the ERA5 divergent flow spectrum is slightly shallower than even $-5 / 3$ throughout this wavenumber range.

Mesoscale motions in the atmosphere are associated with low Rossby number, and almost by definition with comparable magnitudes of rotational and divergent flow KE. For concreteness, if we arbitrarily define a mesoscale threshold wavenumber as that at which the rotational flow KE becomes smaller than $\sqrt{2}$ times the divergent flow KE, then this threshold is at wavenumber $n=87$ for ERA5, 97 for ERAI, 134 for low-res GFS, 203 for JRA-55, and 314 for high-res GFS. It is surprising that these thresholds do not vary monotonically with the nominal resolutions of the analyses, as one might expect if only 
dynamical cascades were in play. The low-res GFS, high-res GFS, and JRA-55 analyses have a relatively high threshold, possibly due to an underrepresented divergent-flow KE, which also results in a steeper mesoscale spectral slope. On the other hand, the threshold for ERAI is at a relatively low wavenumber, and yet ERAI has the steepest mesoscale slope. Clearly, entering the mesoscale wavenumber regime is not always associated with a shallowing of the spectral slope, which suggests that other mechanisms (e.g., scale-selective numerical damping, diabatic heating, and orographic effects) play an important role in determining the mesoscale slope. We consider these effects next.

\section{4) EFFECT OF NUMERICAL DAMPING}

Figure 1a shows large inconsistencies among the analyses in both the mesoscale KE and the mesoscale spectral slopes. All the spectra are steeper than $-5 / 3$ near $n=100$; some are even steeper than -3 . For example, the low-res GFS analysis (black solid line) is steeper than -9 in the $120<n<170$ range.

An obvious candidate explanation for these large differences is the different scale-selective numerical damping (i.e., horizontal diffusion) used in the data-assimilating models. This numerical damping is generally of the form

$$
\frac{\partial \psi}{\partial t}=\cdots \nu \nabla^{p} \psi
$$

for any variable $\psi$, where $\nu$ is the hyperviscosity coefficient and $p$ is the order of the horizontal derivative of $\psi$, which is usually set equal to 2,4 , or higher in various model configurations. In spectral space, this equation is

$$
\frac{\partial \psi_{n}^{m}}{\partial t}=\cdots-\nu\left[\frac{n(n+1)}{a^{2}}\right]^{p / 2} \psi_{n}^{m} .
$$

The coefficient $\nu$ is usually specified such that the damping time scale is a few hours at the largest nominal resolved wavenumber. It is clear from this equation that the numerical damping is strongest at the largest resolved wavenumber and is much weaker at lower wavenumbers. Any such scale-selective numerical damping thus tends to steepen the KE spectral slope. We will revisit this point in section 4 .

\section{5) EFFECT OF BILINEAR INTERPOLATION}

Performing a bilinear interpolation of the analysis winds affects the estimated small-scale KE. The ERAI spectrum, based on winds interpolated to a coarser $0.75^{\circ} \times 0.75^{\circ}$ grid (blue dotted line in Fig. 1a) has lower KE power just below $n=$ 200 than the power computed using a regular F128 Gaussian grid (blue thick solid line), and unrealistically large power above $n=200$ (not shown). On the other hand, interpolating the high-resolution ERA5 winds to a finer $0.25^{\circ} \times 0.25^{\circ}$ grid still causes numerical energy loss at the highest wavenumbers. Because the low-res and high-res GFS analyses were interpolated to $0.5^{\circ} \times 0.5^{\circ}$ and $0.25^{\circ} \times 0.25^{\circ}$ grids, respectively, we suspect some KE loss from bilinear interpolation at the highest wavenumbers in those spectra as well. The KE spectrum from the low-res GFS analysis is cut off at $n=230$, because the bilinear interpolation creates spuriously large KE beyond it.
Despite these caveats, we do not suspect any of the bilinear interpolations performed for this study to affect our results for wavenumbers smaller than $n=170$.

\section{6) KE OF MEAN FLOW VERSUS KE OF TRANSIENTS}

The KE spectra in Fig. 1a discussed thus far are of the total winds (=time mean + transients). The figure also shows the contributions to the total KE in the ERAI reanalysis by the KE of the time-mean winds (MKE; blue thin solid line) and the KE of the transient winds (TKE; blue thin dashed line). Not surprisingly, the MKE spectrum has greatest power at planetary scales $(n \leq 4)$. The TKE spectrum is nearly identical to the total $\mathrm{KE}$ spectrum at synoptic and smaller scales $(n \geq 20)$. For comparison, the MKE spectrum in the higher-resolution ERA5 reanalysis is also shown (green thin solid line). It differs from the ERAI MKE spectrum only at smaller scales $(n>40)$, which account for a very small fraction of the total KE. These total KE versus TKE comparisons confirm that almost all of the KE beyond $n=20$, that is, at synoptic and smaller scales, is associated with the TKE. The mesoscale spectral inconsistencies of the total KE are therefore not due to discrepancies in the MKE spectra.

In summary, Fig. 1a provides an overview of the KE spectra derived from several modern state-of-the-art global atmospheric circulation datasets. The spectra are highly inconsistent at mesoscales, and no spectrum has a precisely $-5 / 3$ slope inferred from aircraft data in early observational studies. We stress that modern analyses utilize many more observations with much better coverage in time and space, and the spherical harmonic expansion method used to derive the global $\mathrm{KE}$ spectrum is also superior to that used in the Nastrom and Gage (1985) and other observational studies. Despite this, the spectra in the modern datasets clearly do not agree. We explore possible reasons for this in section $2 \mathrm{~d}$, and discuss other issues with the analyses in section $2 \mathrm{e}$.

\section{d. Spatial heterogeneity and spectral slopes of $K E$ in subsynoptic/mesoscale wavenumber bands}

Figure 3 shows global maps of KE in two wavenumber bands: band $1(64 \leq n \leq 100)$ and band $2(101 \leq n \leq 158)$, in the low-res GFS analysis, ERAI, and ERA5, as well as their associated regional spectral slopes (explained below). As far as we are aware, such maps have not been previously presented in the meteorological literature, but are clearly relevant to the issue under consideration. It is interesting that although the ERAI and low-res GFS analyses have nearly identical spectral resolutions, band-1 KE in ERAI is much weaker overall than in the low-res GFS analysis, consistent with Fig. 1a, and also much weaker than in the higher-resolution ERA5. We chose band 1 to highlight the KE in subsynoptic scales, partly to assess the extent to which it is determined by downscale cascades from synoptic scales, and indeed band-1 KE in ERAI and ERA5 is strongest in the extratropical regions of large synoptic variability (Figs. 2b,c). However, band-1 KE in the low-res GFS analysis is largest in the tropical convective regions, where it is also larger than in ERAI and ERA5. This is likely a result of the different convective parameterizations used in 
(a) GFS band-1 KE $(n=64-100)$

(b) ERAI band-1 KE $(n=64-100)$

(c) ERA5 band-1 KE $(n=64-100)$

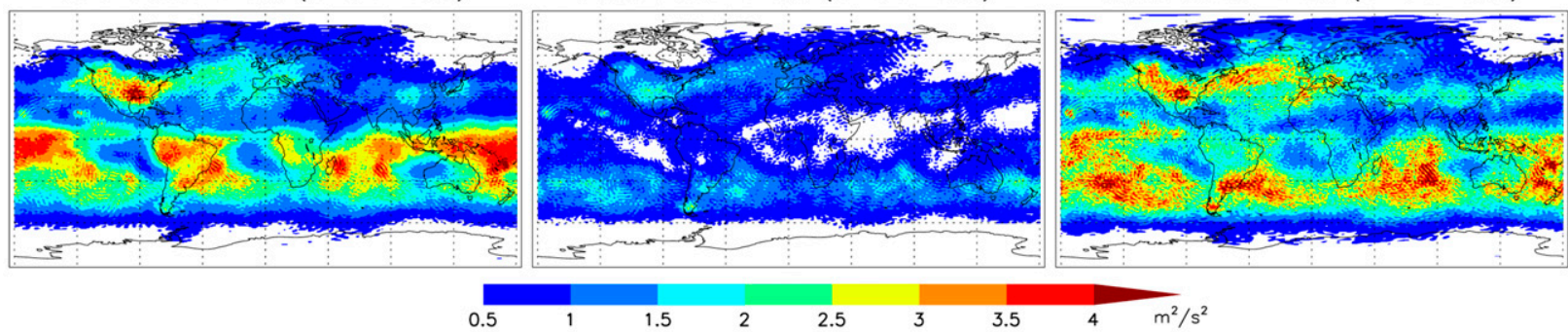

(d) GFS band-2 KE ( $n=101-158)$

(e) ERAI band-2 KE $(n=101-158)$

(f) ERA5 band-2 KE $(n=101-158)$

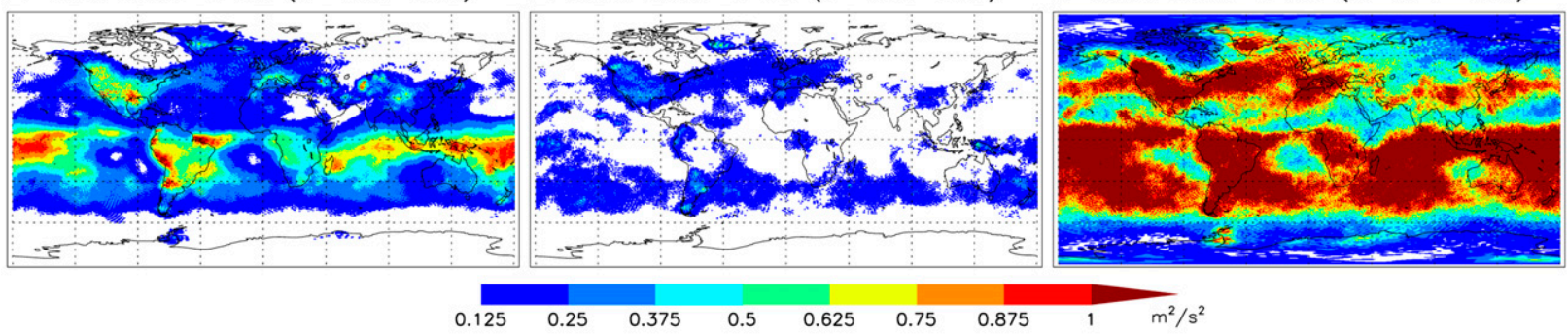

(g) GFS Spctrl Slope $(n=64-158)$

(h) ERAI Spctrl Slope $(n=64-158)$

(i) ERA5 Spctrl Slope $(n=64-158)$
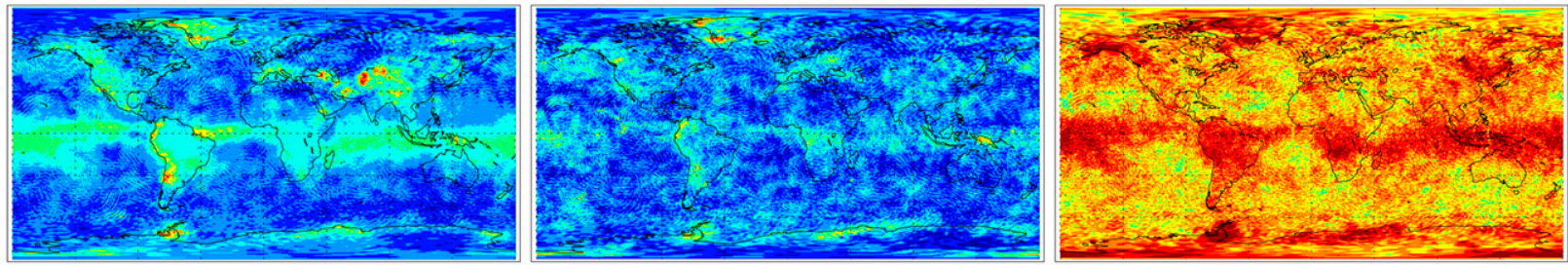

$-6.33$

$-5.67$

FIG. 3. (a)-(c) KE in wavenumber band $1(64 \leq n \leq 100)$ from the low-res GFS analysis, ERAI, and ERA5. (d)-(f) As in (a)-(c), but for $\mathrm{KE}$ in wavenumber band $2(101 \leq n \leq 158)$. (g)-(i) The local mesoscale KE spectral slope $(-\alpha)$ derived from the ratios of band-1 and band-2 KE in (g) the low-res GFS analysis, (h) ERAI, and (i) ERA5.

the models. The discrepancy is striking over the Indo-Pacific oceanic warm pool. The effect of orography on band-1 KE is evident east of the Rockies and somewhat less clearly east of the Andes and the Tibetan Plateau. There are, however, large discrepancies among the three datasets of these orographic effects as well. This is not surprising, considering the different orographic drag parameterizations used in the corresponding data-assimilating models, which have a considerable effect on the atmospheric circulation (Sandu et al. 2019; Elvidge et al. 2019).

Band-2 KE has a similar spatial structure to that of band-1 $\mathrm{KE}$, but much weaker magnitude overall (note the smaller shading interval). The pattern similarity is consistent with regional inertial cascades in bands 1 and 2 generated within spatially varying envelopes of larger-scale variability $(n<$ 20). However, the fact that the spatial structures of the KE in the two bands are also partly determined by spatially varying deep convection and orographic effects demands that we take a closer look. If the results in Fig. 3 were entirely determined by turbulent cascades from large scales, then the ratio of band- 1 and band- $2 \mathrm{KE}$ would be spatially uniform, which is not true. Indeed, one can use the spatially varying ratio to estimate an effective local slope $\alpha$ of the mesoscale spectrum, assuming a power-law spectrum $\sim n^{-\alpha}$, through the expression

$$
\alpha=1-\ln \frac{\sum_{n=\left[n_{*}\right]}^{n=n_{2}} \mathrm{KE}_{n}}{\sum_{n=n_{1}}^{n=\left[n_{*}\right]} \mathrm{KE}_{n}} / \ln \sqrt{\frac{n_{2}}{n_{1}}} .
$$

We specified $n_{1}=64, n_{2}=158$, and $n_{*}=\sqrt{n_{1} n_{2}} \cong 100.56$ in this expression to estimate the effective $\alpha$ over bands 1 and 2 . Figures $3 \mathrm{~g}-\mathrm{i}$ show that the local spectral slopes in ERAI are steeper than -3 nearly everywhere, steeper than in the low-res GFS analysis, and much steeper than in ERA5. Surprisingly, the gentlest slopes are found in tropical convective areas and not in the extratropical regions of large synoptic variability. The slopes are also gentler along the Rockies and Andes mountains. The slopes in ERA5 are between -3.67 and -1.67 over most of the globe, and gentler than $-1.67(=-5 / 3)$ in tropical convective areas, Alaska, and southeast of Lake Baikal. 


\section{2-hour Forecast Convective Precip Rate}

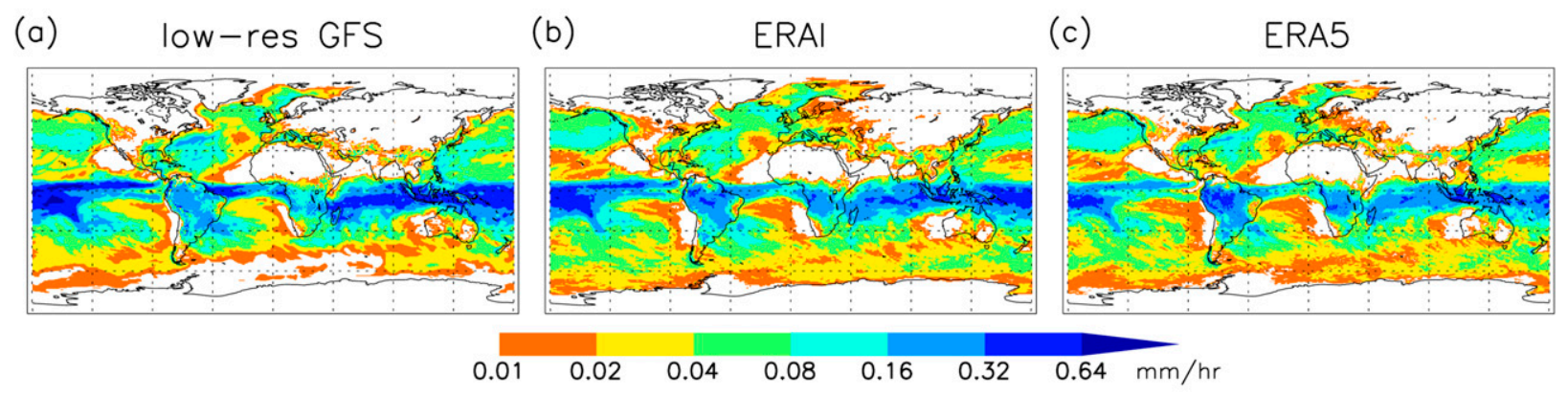

\section{(d) low-res GFS}

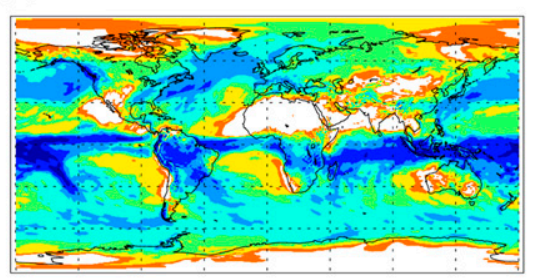

\section{2-hour Forecast Total Precip Rate}

(e)

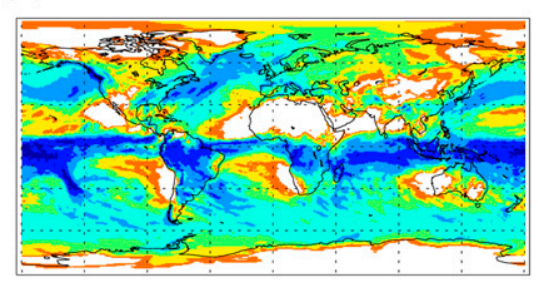

(f)

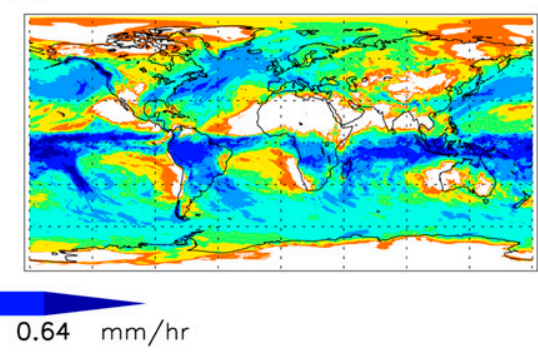

FIG. 4. The average 12-hourly (a)-(c) convective precipitation and (d)-(f) total precipitation rate in the 4 Feb to 14 Mar 2016 period in the (a),(d) low-res GFS, (b),(e) ERAI, and (c),(f) ERA5 datasets.

The spatial pattern of the local slopes in all these datasets is strongly reminiscent of the spatial pattern of convective precipitation, especially in the tropics (Figs. $4 \mathrm{a}-\mathrm{c}$ ). This suggests that the gentler than -3 mesoscale slopes of global KE spectra are a global signature of relatively large mesoscale $\mathrm{KE}$ in areas of strong latent heat release. In such areas the conversion of the eddy potential energy generated by the heating to eddy kinetic energy is strongly affected by the small-scale numerical damping used in models (as also demonstrated clearly in Fig. 9c below). Thus, even though the differences among the convective precipitation rates in the three datasets in Figs. $4 a-c$ are relatively minor, those differences are magnified in the mesoscale KE by the different small-scale numerical damping in the models. The spatial patterns of the total precipitation in the three datasets are shown in Figs. 4d-f. They are similar to the convective precipitation patterns, but with larger magnitudes in extratropical storm-track regions associated with substantial contributions from resolved large-scale precipitation. Because of these contributions, the patterns of the local mesoscale slopes in in Fig. 3 are less similar to those of total precipitation in Fig. 4 in the extratropics than they are to the patterns of convective precipitation in the tropics.

The spatial patterns of KE magnitudes and local spectral slopes in a higher $101 \leq n \leq 169$ wavenumber range (not shown) are similar to those in the $64 \leq n \leq 158$ range shown in Fig. 3, but with generally smaller KE magnitudes and steeper spectral slopes. Overall, these features of the low-res GFS, ERAI, and ERA5 datasets are inconsistent with the NastromGage spectrum, and their obvious spatial heterogeneity is also inconsistent with theories of homogenous and isotropic largescale atmospheric turbulence.

\section{e. Credibility of analyses at mesoscales}

One might question the credibility of the analyses used here given their inconsistencies. We stress again that these inconsistencies occur mainly at subsynoptic and smaller scales that account for a small portion of the total KE. On larger scales, the analyses agree well with each other (e.g., Harada et al. 2016; Long et al. 2017) and with observations (Slivinski et al. 2019; Hersbach et al. 2020). Note that since such analyses represent a compromise between observations and forecast guesses and take into account the expected errors of each, their expected errors are smaller than those of both the observations and the guesses. Despite this, the analyses obviously do not agree at small scales. We consider several potential sources of their discrepancies below.

\section{1) ISSUES WITH OBSERVATIONS AND DATA ASSIMILATION}

Modern analyses, including those used in this study, assimilate the same types of wind and temperature observations that have been used in purely observation-based KE spectrum studies (e.g., Frehlich and Sharman 2010), and many other types of observations as well. For example, in addition to abundant satellite observations, nearly 400000 ACARS/AMDAR, METAR, and other conventional upper-air wind observations were assimilated every day in the ERAI and ERA5 of 2016 (see Fig. 4 of Hersbach et al. 2020). The beneficial 
impact of assimilating such observations on global weather forecasts has been well documented (e.g., Petersen 2016).

Given the similar numbers and types of observations used in the ERAI, ERA5, JRA-55, and GFS analyses, discrepancies among their relatively small mesoscale $\mathrm{KE}$ in spectral bands 1 and 2 (typically between $10^{-1}$ and $10^{-3} \mathrm{~m}^{2} \mathrm{~s}^{-2}$; see Fig. 1a) arise from discrepancies in the way those observations are assimilated (e.g., through different specified observation error covariances) as well as in the background guess forecasts (e.g., through different physical parameterizations and scale-dependent damping). For example, in some analyses the aircraft observations are thinned when correlated representativeness errors become an issue (Cardinali et al. 2003). Second, scale-dependent balance constraints imposed on the analyses tend to reduce the magnitude of divergent mesoscale motions. Third, an inefficient elimination of spatially correlated background guess errors by the analysis algorithm can result in spurious spatial covariances in the analyses (Hollingsworth and Lonnberg 1989). Spatial covariances are closely related to wavenumber spectra, and because both they and the spectra decrease in magnitude with wavenumber, an inefficient elimination of the spatially correlated parts of the background errors can cause erroneous steepening of the mesoscale spectral slope in the analyses. Finally, as indicated in Table 1, all the analyses used here (except the low-res GFS analysis) are actually performed at a lower resolution than the nominal resolution indicated in the final analysis product. The analysis spectra at the high wavenumber end of the nominal resolution are thus not directly constrained by observations.

\section{2) EFFECTIVE RESOLUTION OF THE ANALYSES}

Model developers are well aware of the inaccurate and even some unphysical aspects of their models near the limit of their nominal resolutions. As a rule of thumb, for nominal model grid sizes $\Delta x$, they often estimate the effective grid sizes to be $4 \Delta x, 6 \Delta x$, or even as large as $8 \Delta x$ (partly justifying these estimates, it must be mentioned, to correspond to wavenumbers beyond which the model spectral slope becomes steeper than $-5 / 3$ ). In our context, using $8 \Delta x$ implies a credible effective highest wavenumber $n_{\text {eff }}$ of only 384 for the high-res GFS analyses and forecasts, 160 for ERA5, 80 for JRA-55, and 64 for ERAI and the low-res GFS analyses. These effective resolutions are much more conservative than those estimated in section $2 \mathrm{c}(1)$, although the effective resolutions of the highres GFS analyses and forecasts and ERA5 remain higher than 158 even by this criterion. It is interesting that the high-res GFS analyses and forecasts have a higher effective resolution and a much better-resolved mesoscale wavenumber regime than ERA5, and yet have steeper spectral slopes than ERA5 in the $64 \leq n \leq 158$ wavenumber range. The slopes are also steeper than $-5 / 3$. Should one conclude from this that the high-res GFS spectra are "wrong"? The answer would be "yes" if one assumes the "truth" is known (e.g., a $-5 / 3$ mesoscale slope), but given the importance of the large and uncertain diabatic contributions to the spectrum highlighted in this study, one cannot regard that truth as settled. We also note that defining the effective model or analysis resolution to be the wavenumber $n_{\text {eff }}$ beyond which the spectral slopes start to deviate from the $-5 / 3$ mesoscale slope (e.g., Skamarock 2004) effectively assumes that the true slope is $-5 / 3$. By this definition, the high-res GFS analyses and forecasts would have no mesoscale wavenumber regimes, which is clearly not true.

\section{3) Spatial heterogeneity of the mesoscale KE}

The spatial heterogeneity of the mesoscale KE and regional spectral slopes is a robust feature of all the analyses examined here, and is actually consistent with previous observational studies, although this aspect is not highlighted in those studies. For example, the results in Frehlich and Sharman (2010) show a clear dependence of the mesoscale spectral slope on latitude, season, topography, and altitude (see their Figs. 9-14 and Tables A1-A10), as implied by structure functions derived from rawinsonde and aircraft observations at separation distances $s$ between 400 and $20 \mathrm{~km}$. Li and Lindborg (2018) argued that at least 1000 flight segments were needed to obtain reasonably converged ratios of the divergent and rotational wind energy spectra. Although they sought a statistical sampling explanation for their inhomogeneous results, a simpler explanation from our perspective is that the divergent and rotational flow statistics are actually inhomogeneous over the globe. In fact, despite their claim that "there is no dramatic difference between land and ocean," Li and Lindborg (2018) actually found, even after averaging over more than 2000 upper-tropospheric flight segments, that the mesoscale divergent flow energy over land is approximately $40 \%$ larger than over the oceans (see their Fig. 10). This again indicates the heterogeneous nature of the flow statistics. Thus, regardless of whether the heterogeneity arises from convective or orographic effects (e.g., Hamilton et al. 2008), upwardpropagating gravity waves (e.g., Li and Lindborg 2018), or other mechanisms, its existence itself is robust across observations, models, and the analysis datasets used here. Our results strongly suggest that the heterogeneity is associated with latent heat release, as evident from the striking resemblance of the spatial pattern of the convective precipitation rate in Fig. 4 to that of the spectral slopes in Fig. 3 in our datasets. In this important broader sense, the analyses used here are consistent and credible even at mesoscales.

\section{Sensitivity of the KE spectrum to stochastic physics}

The important influence of latent heat release on the mesoscale KE spectrum motivated us to explore the sensitivity of the spectrum to changes in model physics. To this end we generated four parallel sets of 80-member ensembles of 10-day forecasts for 80 forecast cases in the 4 February to 14 March 2016 period, using the low-res GFS forecast model and low-res GFS analyses as initial conditions. The sets differed in their use of stochastic parameterizations (SPs) of the model's diabatic tendencies, whose generally beneficial forecast impacts were reported in Wang et al. (2019). Table 2 lists the four sets of ensemble forecasts performed:

1) NoSP forecasts: Without stochastic parameterizations

2) Control forecasts: With stochastically perturbed physical tendencies (SPPT; Palmer et al. 2009; Shutts et al. 2011) 
TABLE 2. List of low-res GFS forecast sets.

\begin{tabular}{|c|c|c|c|}
\hline Expt & Stochastic parameterizations (SP) & SP perturbation scale length $(L)$ & Hyperviscosity coefficient \\
\hline noSP & None & - & $\nu$ \\
\hline Control $_{\mathrm{L} 100}$ & SPPT + SHUM & $100 \mathrm{~km}$ & $\nu$ \\
\hline Control & SPPT + SHUM & $500 \mathrm{~km}$ & $\nu$ \\
\hline $\mathrm{SP}_{\text {full }}$ & SPPT + SHUM + SKEB & $500 \mathrm{~km}$ & $\nu$ \\
\hline $\mathrm{SP}_{\text {half }}$ & SPPT + SHUM + SKEB & $500 \mathrm{~km}$ & $0.5 \nu$ \\
\hline
\end{tabular}

plus boundary layer stochastic humidity (SHUM) parameterizations (Tompkins and Berner 2008), both with a specified horizontal length scale $L=500 \mathrm{~km}$ of the stochastic perturbations

3) Control $_{\mathrm{L} 100}$ forecasts: As in the Control forecasts, but with a specified length scale $L=100 \mathrm{~km}$ of the stochastic perturbations

4) $\mathrm{SP}_{\text {full }}$ forecasts: As in the Control forecasts, but with additional stochastic kinetic energy backscatter (SKEB; Shutts 2005; Berner et al. 2009, 2012, 2015) parameterizations with a specified length scale $L=500 \mathrm{~km}$ of the stochastic perturbations

The NoSP and Control forecasts were the same as described in Wang et al. (2019), except the forecast length considered here is 10 days. Note that there were $80 \times 80=6400$ ten-day forecasts in each of these four forecast sets.

Figure 5 compares the mean of the 6400 day- 10 KE spectra in each forecast set with the mean of the $6400 \mathrm{KE}$ spectra in the verifying low-res GFS analyses. The results are shown as both differences and ratios. All the day- 10 forecast spectra are lower and steeper than the analysis spectra on synoptic and shorter scales $(n \geq 20)$. Comparing the Control (green) and NoSP (blue) spectra shows the impact of the SPPT + SHUM parameterizations. There is a discernible impact on synoptic and larger scales, but an apparently negligible impact on mesoscales. This is not surprising, given that the mesoscale $\mathrm{KE}$ is at least an order of magnitude smaller than the synoptic-scale $\mathrm{KE}$. Indeed, the KE summed over $n=1$ to $n=80\left(\mathrm{KE}_{n=1-80}\right)$ accounts for more than $99.9 \%$ of the KE summed over $n=1$ to $n=170\left(\mathrm{KE}_{n=1-170}\right)$ in the GFS analyses (Fig. 6). The largescale $\mathrm{KE}$ accounts for an even larger fraction of the total $\mathrm{KE}$ in the day-10 forecasts (i.e., $\mathrm{KE}_{n=1-80}>99.9 \%$ of $\mathrm{KE}_{n=1-170}$ ), because the mesoscale $\mathrm{KE}$ is more strongly damped than the large-scale KE by the model's scale-dependent horizontal diffusion.

The larger impact of the SPs on larger scales is possibly due to the relatively large specified SP length scale $(L=500 \mathrm{~km})$ in the Control and $\mathrm{SP}_{\text {full }}$ forecasts. This corresponds to stochastic diabatic forcing at approximately $n=40$, which possibly impacts larger scales via an inverse energy cascade hypothesized by Lilly (1983). The impact, however, is not of the same sign at all wavenumbers lower than $n=40$. For example, the Control forecasts have larger $\mathrm{KE}$ at $n \leq 7$ and smaller $\mathrm{KE}$ in the range $8 \leq n \leq 20$ than the NoSP forecasts, which suggests a more complex multiscale interaction than a simple inverse cascade. Using additional SKEB parameterizations in the $\mathrm{SP}_{\text {full }}$ forecasts hardly affects this wavenumber dependence. Specifying a shorter SP length scale $L=100 \mathrm{~km}$ (corresponding to $n=200$ ) in the $\mathrm{Control}_{\mathrm{L} 100}$ forecasts does generate more mesoscale $\mathrm{KE}$, but again the impact on larger scales is very similar.

Figure 7 provides a regional perspective of the impact on $\mathrm{KE}$ in the $64 \leq n \leq 170$ wavenumber range by the stochastic parameterizations in the Control and $\mathrm{Control}_{\mathrm{L} 100}$ forecasts. It is interesting, and important, that the impact is sensitive to the specified length scale $L$ of the stochastic perturbations. Specifying $L=500 \mathrm{~km}$ in the control forecasts does not ubiquitously increase the KE in this range (Fig. 7a); indeed, in many tropical convective regions such as the Indo-Pacific, it causes a decrease. On the other hand, specifying a shorter scale $L=100 \mathrm{~km}$ in the Control $_{\mathrm{L} 100}$ forecasts does lead to a pervasive increase of KE in this range in the tropics (Fig. 7b). Note that the low-res GFS analyses were generated using

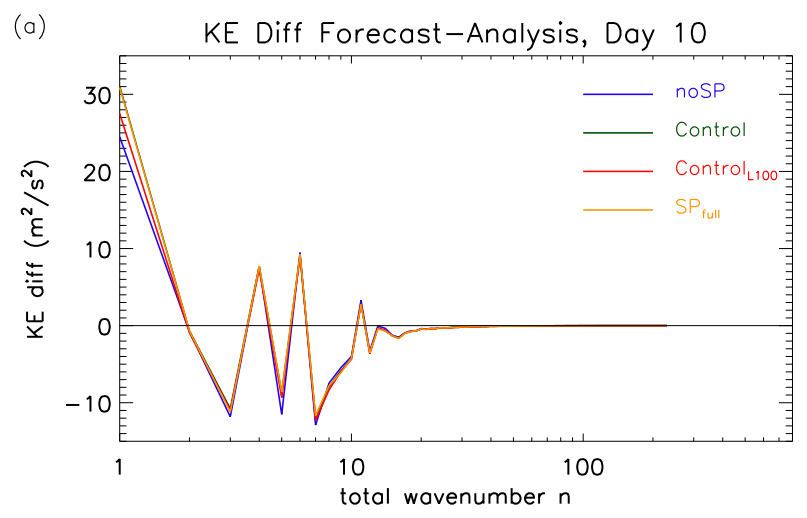

(b)

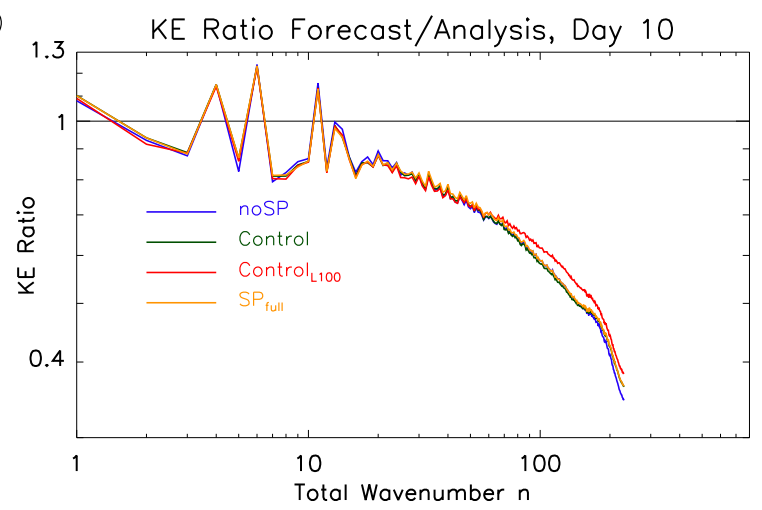

FIG. 5. (a) Differences of the mean KE spectra obtained in four sets of 10-day low-res GFS model forecasts with various implementations of stochastic physics parameterizations from the mean spectrum of the verifying low-res GFS analyses and (b) the ratios of the forecast and analysis spectra. See text for detailed descriptions of the four forecast experiments. 


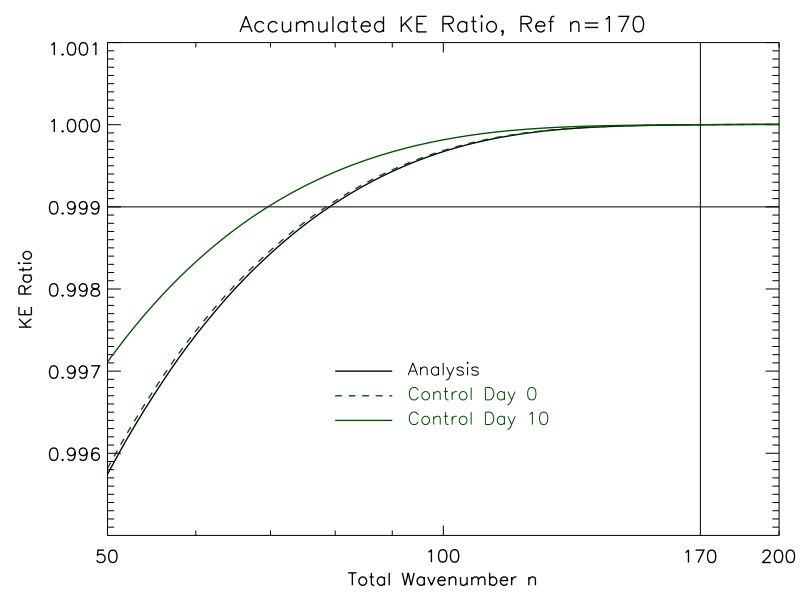

FIG. 6. Ratio of KE summed over wavenumbers 1 to $n$ to KE summed over wavenumbers 1 to 170 in the low-res GFS analysis (black solid line), Control forecast on day 0 (green dash line), and Control forecast on day 10 (green solid line). Wavenumber $n=170$ is the effective resolution of the low-res GFS. Control forecast on day 0 refers to the model output after the first time step ( $20 \mathrm{~min})$, so is slightly different from the analysis.

$L=500 \mathrm{~km}$, whose impact on the band-2 KE in Fig. 3, judging by Fig. $7 \mathrm{a}$, is likely negative over the tropical Indo-Pacific. Despite this, the band-2 KE in this region in Fig. 3, as already mentioned, is larger in the low-res GFS analyses than in the ERAI and ERA5 analyses. It is conceivable that low-res GFS analyses regenerated using $L=100 \mathrm{~km}$ might show even larger $\mathrm{KE}$ in band 2, judging by Fig. 7c. We have not performed such a regeneration, partly because of uncertainty in the choice of $L$. It is important to reduce this uncertainty, given the sensitivity of the mesoscale KE to the value of $L$.

\section{Sensitivity of the KE spectrum to numerical damping}

An intuitive explanation of the lower subsynoptic KE strength and steeper spectral slopes of the day-10 forecast spectra in Fig. 5 than the analysis spectra is the relatively large numerical damping at high wavenumbers in the forecast model. Such a damping is usually implemented in models to control numerical stability, but its effect on the KE spectrum needs to be carefully considered. To estimate this effect, we generated another 80-member ensemble of 10-day low-res GFS forecasts ("SP ${ }_{\text {half }}$ ") initialized with the same low-res GFS analyses as the other forecast ensembles, and compared it with the $\mathrm{SP}_{\text {full }}$ ensemble. The only difference between the $\mathrm{SP}_{\text {full }}$ and $\mathrm{SP}_{\text {half }}$ forecasts was that the former used the default value of the eighth-order hyperviscosity coefficient, whereas the latter used half of the default value.

Figure 8 compares the $\mathrm{KE}$ spectra in the $\mathrm{SP}_{\text {full }}$ and $\mathrm{SP}_{\text {half }}$ forecasts with the spectra in the verifying low-res GFS analyses, in a similar format to Fig. 5. Results are shown for both day-1 and day-10 spectra. The reduced damping in $\mathrm{SP}_{\text {half }}$ clearly enhances the mesoscale $\mathrm{KE}(100<n<200)$ and reduces the steep mesoscale spectral slope (Fig. $8 \mathrm{~b}$ ). This effect is already discernible by day 1 , and persists through day 10 of the (a)

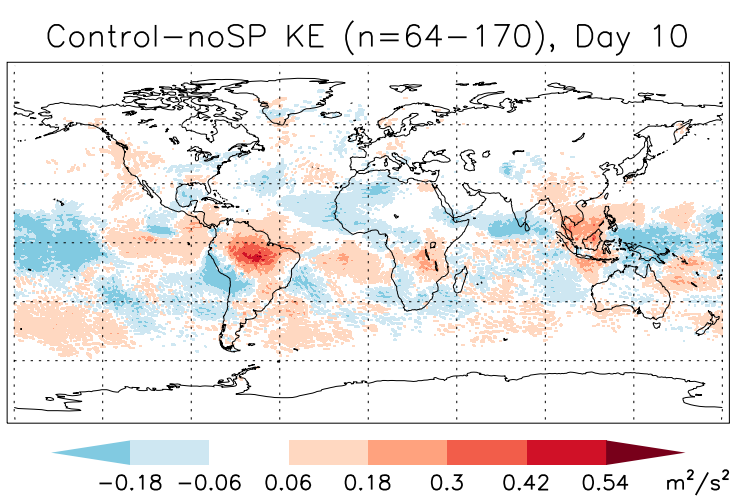

(b)

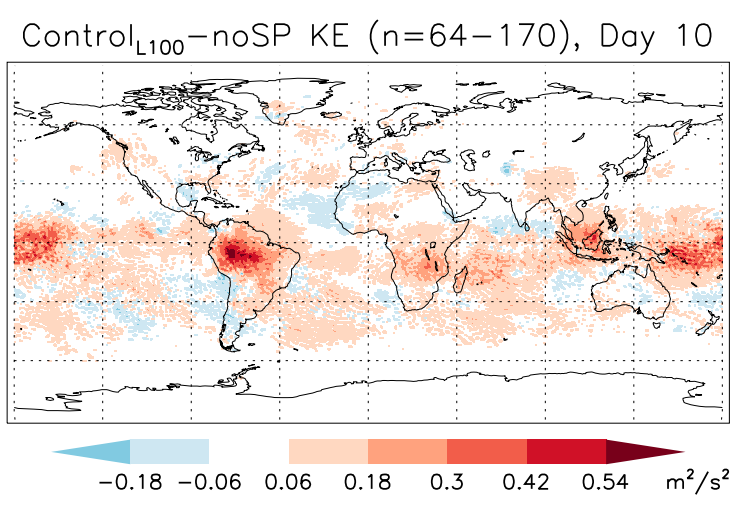

(c)

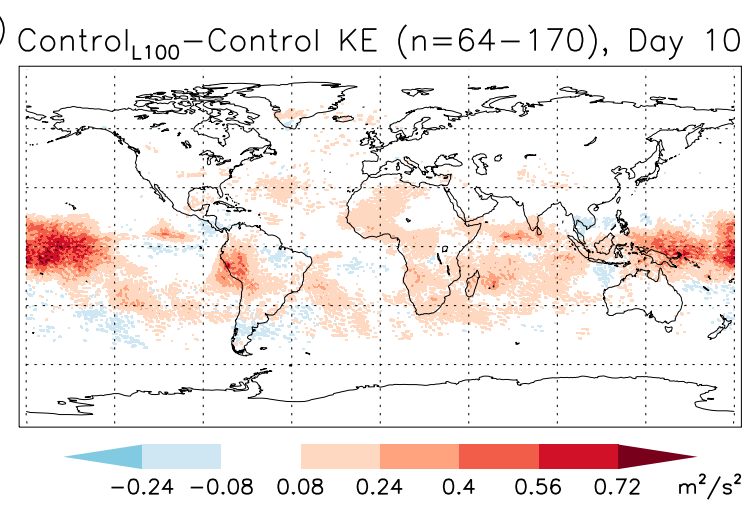

FIG. 7. Difference of KE in wavenumber band 1 ( $64 \leq n \leq 100)$ between (a) Control and NoSP, (b) Control $_{\mathrm{L} 100}$ and NoSP, and (c) Control $\mathrm{L} 100_{\text {and }}$ Control forecasts on day 10. The Control and Control $_{\mathrm{L} 100}$ forecasts include stochastic parameterizations with prescribed stochastic perturbation length scales of 500 and $100 \mathrm{~km}$, respectively. The NoSP forecasts have no stochastic parameterizations. See text for a full explanation.

forecasts. Indeed, by day 1 the $\mathrm{KE}$ in the $\mathrm{SP}_{\text {half }}$ near $n=170$ is even higher than in the verifying low-res GFS analyses. Nonetheless, the fact that the ratio of the $\mathrm{SP}_{\text {half }}$ to analysis spectral $\mathrm{KE}$ is not uniform on mesoscales shows that the forecast mesoscale spectrum cannot be made identical to the analysis spectrum merely by tuning the hyperviscosity coefficient. Still, the sensitivity is clear.

Figure 8 also shows the mean day- 1 and day- $10 \mathrm{KE}$ spectra in the 80 high-res (T1534) GFS forecasts, relative to the mean 
(a)

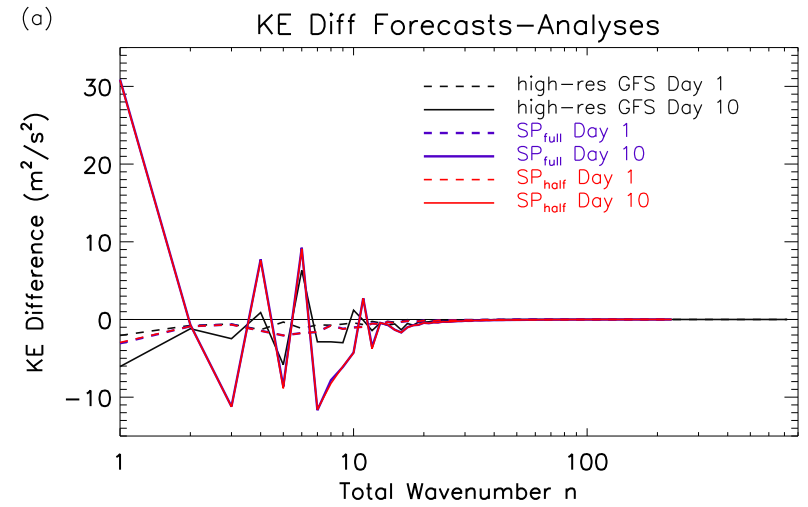

(b)

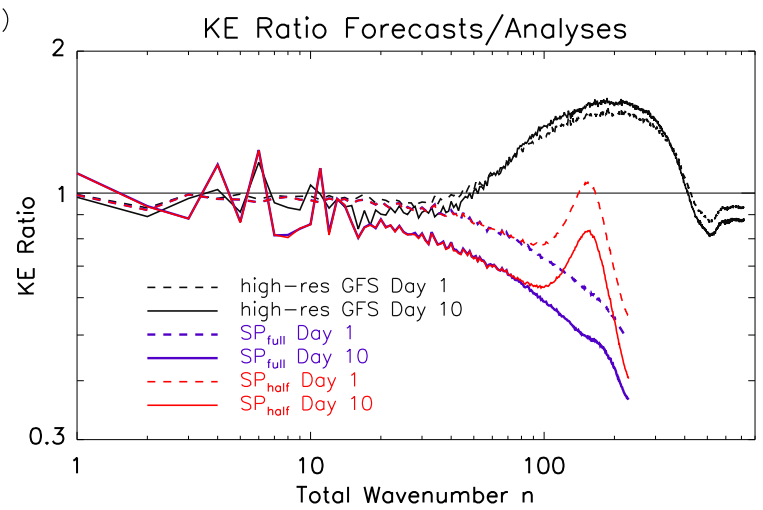

FIG. 8. (a) Differences and (b) ratios of the mean spectra in GFS forecasts and verifying GFS analyses for day 1 (dashed) and day 10 (solid) forecasts. Results are shown for high-res forecasts (black), low-res forecasts using the full default value of the hyperviscosity coefficient (blue), and low-res forecasts using half the default value (red). Note that the verifying analyses for the high-res forecasts are the high-res GFS analyses, and for the low-res forecasts are the low-res GFS analyses.

spectrum in the verifying high-res GFS analyses, in the 4 February to 14 March 2016 period. The high-res model uses a much smaller hyperviscosity coefficient of the eighth-order hyperdiffusion, but is otherwise nearly identical to the low-res (T254) GFS model. We note again that the precise value of such a damping coefficient in models is dictated by numerical stability rather than by physical considerations. With this in mind, the high-res results in Fig. 8 are also revealing. They show that the mesoscale KE and spectral slopes in the high-res forecasts are higher than even in the high-res analyses. This effect is already clear by day 1 , which suggests that the damping coefficient in the high-res GFS model is too small. This also suggests that the higher mesoscale KE and less steep slope of the high-res GFS analysis spectrum in Fig. 1a, relative to the low-res GFS analysis spectrum, is likely due to the same relatively small damping coefficient used in the high-res analysis system. Despite this, the high-res GFS analyses and day-10 forecast spectra are still lower and steeper than the lower resolution ERA5 spectrum, as already noted.

Figure 9 provides a regional perspective of the day-10 KE biases in the $101 \leq n \leq 170$ wavenumber range of the low-res (o)

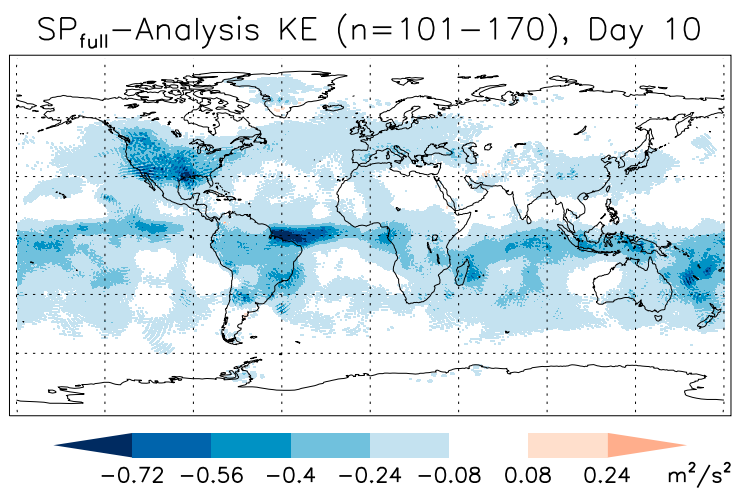

(b)

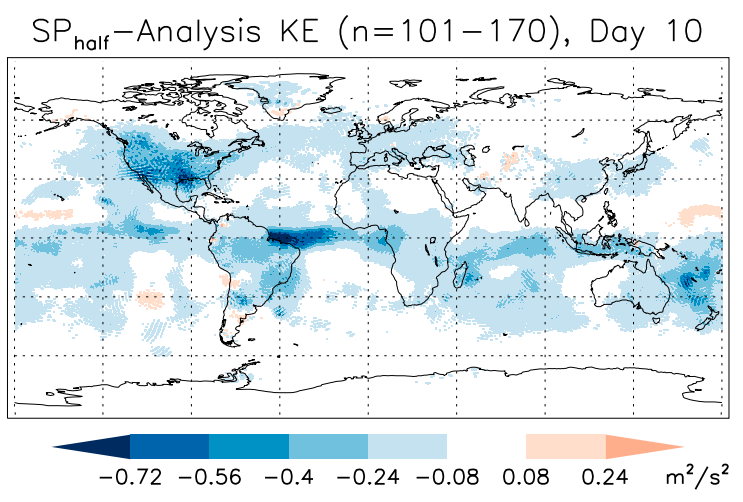

(c)

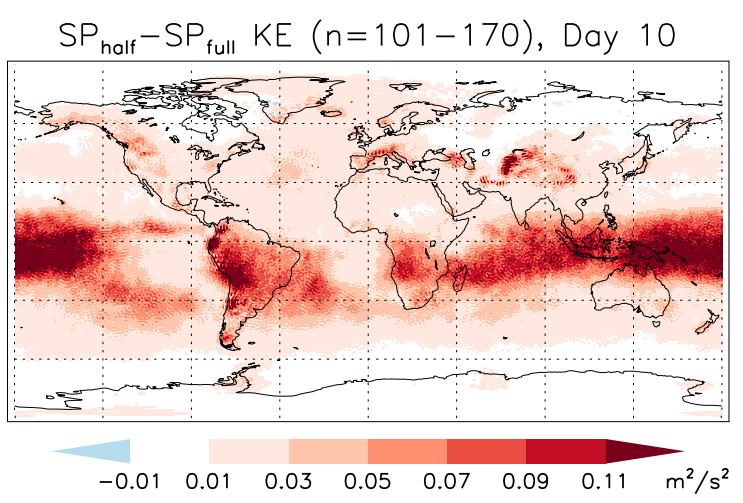

FIG. 9. Difference of KE in the wavenumber band $101 \leq n \leq 170$ between (a) the $\mathrm{SP}_{\text {full }}$ forecasts (using the full default value of the hyperviscosity coefficient) and the low-res GFS analyses, (b) the $\mathrm{SP}_{\text {half }}$ forecasts (using half the default value of the hyperviscosity coefficient) and the low-res GFS analyses, and (c) the $\mathrm{SP}_{\text {half }}$ and $\mathrm{SP}_{\text {full }}$ forecasts. Note that the color shading interval in (c) is different from that in (a) and (b).

$\mathrm{SP}_{\text {full }}$ and $\mathrm{SP}_{\text {half }}$ forecasts relative to the $\mathrm{KE}$ in the verifying low-res GFS analyses. The biases are negative over nearly the entire globe in both cases, consistent with the numerical damping in the low-res model being too strong in this wavenumber range. Using the reduced damping in the $\mathrm{SP}_{\text {half }}$ forecasts reduces this negative bias everywhere, as evident from the bias difference map in Fig. 9c. The positive impact of the reduced damping is largest in tropical convective regions; indeed, the impact map in Fig. 9c is again strongly reminiscent of the 
mean convective precipitation map in Fig. 4. Recalling that the global mesoscale spectral slopes in Fig. 1a are strongly influenced by the mesoscale $\mathrm{KE}$ in tropical convective regions in Fig. 3, the results in Fig. 8 suggest how spectrum differences among different analysis datasets may also arise from differences in the numerical damping, and not just the convective heating parameterizations implemented in the data-assimilating models.

\section{Summary and concluding remarks}

This study has revealed large inconsistencies in global upper-tropospheric mesoscale $(n>100) \mathrm{KE}$ spectra deduced from several modern global wind datasets, of as much as a factor of 4 at $n=100$ and a factor of 47 at $n=170$. The spectra also generally have steeper than $-5 / 3$ slopes at these scales, inconsistent with simple theories and numerical models of adiabatic turbulent cascades and also with early observational estimations from aircraft data. We traced the sources of the inconsistencies among the modern datasets to inconsistences in the mesoscale $\mathrm{KE}$ in areas of large latent heat release and near orography. The mesoscale KE in such regions, especially in the tropics, makes a large contribution to the global mesoscale KE, increasing its magnitude and shallowing its spectral slope. This suggests that the relatively shallow slope seen in the high-res GFS and ERA5 spectra in Fig. 1a is in fact largely due to this diabatic contribution, modulated by damping, rather than due to presumably better-resolved adiabatic turbulent cascades.

We showed that the mesoscale $\mathrm{KE}$ in areas of large latent heat release is sensitive in the NCEP GFS model to stochastic parameterizations of model physics and to the specified hyperviscosity coefficient. There may be similar sensitivities to other aspects of model physics, which may be worth exploring in this as well as other models.

The lack of consensus on the mesoscale KE spectral slope in modern global datasets, and its sensitivity to model physics and numerical damping in the data-assimilating models, suggest that a precise determination of the global mesoscale spectrum remains elusive at present. Perhaps this should not come as a surprise, considering that the $\mathrm{KE}$ in the wavenumber band $81<n<170$ accounts for only $0.1 \%$ of the total KE in the atmosphere, so one is focusing on details rather than the big picture. Nonetheless, it is surprising that the vastly greater numbers of observations used in modern data-assimilation systems are apparently still not appropriately assimilated to "correct" the inevitable errors in model-generated guess fields at these small scales.

The sensitivity of the mesoscale KE spectrum to model physics and numerical damping is known in the modeling community, especially in extratropical modeling contexts. However, its spatial variation over the entire globe appears not to have been examined or documented until this study. In particular, the large impact on the global mesoscale KE spectrum from the mesoscale $\mathrm{KE}$ in tropical convective areas appears not to have been appreciated. Previous studies that investigated the impact of stochastic parameterizations focused on the global KE spectrum (e.g., Berner et al. 2009; Sanchez et al. 2014; Leutbecher et al. 2017) and did not consider the contributions to it from different geographical areas.
They also implied that models can be tuned to agree with the observed spectrum. An important conclusion from our study, however, is that there is as yet no clear "target" global spectrum to tune toward, given the large contribution to the global spectrum from uncertain physics in tropical convective areas.

Despite some evidence from previous studies that the mesoscale spectral slope in observations and models depends on geographical location (e.g., Nastrom and Gage 1985; Li and Lindborg 2018), model resolution and numerical damping (e.g., Terasaki and Tanaka 2009), topographic variation and convection scheme (e.g., Skamarock et al. 2014), and to some extent even on the time integration scheme (Dueben et al. 2020), most high-resolution modeling studies continue to seek a pure $-5 / 3$ mesoscale slope associated with adiabatic cascades. In this regard, the steeper than $-5 / 3$ slopes of the high-res GFS analyses and day-10 forecasts (T1534 nominal resolution) in Fig. 1a provide a counterexample and a reminder that the mesoscale spectrum is not determined only by adiabatic cascades. There are clearly other important contributions to the spectrum, but unfortunately no clear consensus as yet on the magnitudes of those contributions, as highlighted in this study.

In summary, there are large uncertainties in the global mesoscale KE spectrum in modern global analysis datasets that are largely associated with inconsistencies in representing spatially varying diabatic and frictional processes in the data-assimilating models. Given the important impact of such processes on the mesoscale spectral slope, a focus on obtaining a $-5 / 3$ mesoscale slope predicted by simple theories and numerical models of adiabatic turbulent cascades is unwarranted. With their restricted focus on adiabatic dynamics, such theories and simple models can only serve as limited guides for understanding and reproducing the complex details of the mesoscale variability and energy spectrum of the real atmosphere.

Acknowledgments. This research was supported by the Physical Sciences Laboratory of NOAA's Earth System Research Laboratories. We thank Dale Durran of the University of Washington, who provided constructive comments on this research. Special thanks to David Stepaniak of NCAR and to Gilbert Compo and Laura Slivinski of the University of Colorado Boulder for providing detailed information on reanalysis datasets. Computing was performed on NOAA's Remotely Deployed High-Performance Computing Systems. Thoughtful comments and suggestions from the reviewers resulted in significant improvements to the paper. The scientific results and conclusions, as well as any views or opinions expressed herein, are those of the authors and do not necessarily reflect the views of NOAA or the Department of Commerce.

\section{REFERENCES}

Berner, J., G. Shutts, M. Leutbecher, and T. Palmer, 2009: A spectral stochastic kinetic energy backscatter scheme and its impact on flow-dependent predictability in the ECMWF Ensemble Prediction System. J. Atmos. Sci., 66, 603-626, https://doi.org/10.1175/2008JAS2677.1. 
_ T. Jung, and T. N. Palmer, 2012: Systematic model error: The impact of increased horizontal resolution versus improved stochastic and deterministic parameterizations. J. Climate, 25, 4946-4962, https://doi.org/10.1175/JCLI-D-11-00297.1.

—, K. R. Fossell, S.-Y. Ha, J. P. Hacker, and C. Snyder, 2015: Increasing the skill of probabilistic forecasts: Understanding performance improvements from model-error representations. Mon. Wea. Rev., 143, 1295-1320, https://doi.org/10.1175/ MWR-D-14-00091.1.

Bloom, S. C., L. L. Takacs, A. M. da Silva, and D. Ledvina, 1996: Data assimilation using incremental analysis updates. Mon. Wea. Rev., 124, 1256-1271, https://doi.org/10.1175/15200493(1996)124<1256:DAUIAU>2.0.CO;2.

Buehner, M., J. Morneau, and C. Charette, 2013: Four-dimensional ensemble-variational data assimilation for global deterministic weather prediction. Nonlinear Processes Geophys., 20, 669682, https://doi.org/10.5194/npg-20-669-2013.

Cardinali, C., L. Isaksen, and E. Andersson, 2003: Use and impact of automated aircraft data in a global 4DVar data assimilation system. Mon. Wea. Rev., 131, 1865-1877, https://doi.org/ 10.1175//2569.1.

Cho, J. Y. N., and Coauthors, 1999a: Horizontal wavenumber spectra of winds, temperature, and trace gases during the Pacific Exploratory Missions: 1. Climatology. J. Geophys. Res., 104, 5697-5716, https://doi.org/10.1029/98JD01825.

_, R. Newell, and J. D. Barrick, 1999b: Horizontal wavenumber spectra of winds, temperature, and trace gases during the Pacific Exploratory Missions: 2. Gravity waves, quasi-twodimensional turbulence, and vortical modes. J. Geophys. Res., 104, 16 297-16308, https://doi.org/10.1029/1999JD900068.

Dee, D. P., and Coauthors, 2011: The ERA-Interim reanalysis: Configuration and performance of the data assimilation system. Quart. J. Roy. Meteor. Soc., 137, 553-597, https://doi.org/ 10.1002/qj.828.

Dole, R. M., and Coauthors, 2018: Advancing science and services during the 2015/16 El Niño: The NOAA El Niño Rapid Response field campaign. Bull. Amer. Meteor. Soc., 99, 9751001, https://doi.org/10.1175/BAMS-D-16-0219.1.

Dueben, P. D., N. Wedi, S. Saarinen, and C. Zeman, 2020: Global simulations of the atmosphere at $1.45 \mathrm{~km}$ grid-spacing with the Integrated Forecasting System. J. Meteor. Soc. Japan, 98, 551572, https://doi.org/10.2151/jmsj.2020-016.

Elvidge, A. D., and Coauthors, 2019: Uncertainty in the representation of orography in weather and climate models and implications. J. Adv. Model. Earth Syst., 11, 2567-2585, https:// doi.org/10.1029/2019MS001661.

Frehlich, R., and R. Sharman, 2010: Climatology of velocity and temperature turbulence statistics determined from rawinsonde and ACARS/AMDAR data. J. Appl. Meteor. Climatol., 49, 1149-1169, https://doi.org/10.1175/2010JAMC2196.1.

Hamilton, K., Y. O. Takahashi, and W. Ohfuchi, 2008: Mesoscale spectrum of atmospheric motions investigated in a very fine resolution global general circulation model. J. Geophys. Res., 113, D18110, https://doi.org/10.1029/2008JD009785.

Harada, Y., and Coauthors, 2016: The JRA-55 Reanalysis: Representation of atmospheric circulation and climate variability. J. Meteor. Soc. Japan, 94, 269-302, https://doi.org/ 10.2151/jmsj.2016-015.

Hersbach, H., and Coauthors, 2018: Operational global reanalysis: Progress, future directions and synergies with NWP. ECMWF ERA Rep. 27, 65 pp., https://doi.org/10.21957/tkic6g3wm.

_ and Coauthors, 2020: The ERA5 global reanalysis. Quart. J. Roy. Meteor. Soc., 146, 1999-2049, https://doi.org/10.1002/qj.3803.
Hollingsworth, A., and P. Lonnberg, 1989: The verification of objective analyses: Diagnostics of analysis system performance. Meteor. Atmos. Phys., 40, 3-27, https://doi.org/ 10.1007/BF01027466.

Huang, X.-Y., and P. Lynch, 1993: Diabatic digital-filtering initialization: Application to the HIRLAM model. Mon. Wea. Rev., 121, 589-603, https://doi.org/10.1175/1520-0493(1993) $121<0589:$ DDFIAT $>2.0 . C O ; 2$.

Ji, M., and Coauthors, 2016: Dynamical core evaluation test report for NOAA's Next Generation Global Prediction System (NGGPS). NOAA Rep., 95 pp., https://repository.library.noaa.gov/view/ noaa/18653.

Judt, F., 2018: Insights into atmospheric predictability through global convection-permitting model simulations. J. Atmos. Sci., 75, 1477-1497, https://doi.org/10.1175/JAS-D-17-0343.1.

Kitamura, Y., and Y. Matsuda, 2010: Energy cascade processes in rotating stratified turbulence with application to the atmospheric mesoscale. J. Geophys. Res., 115, D11104, https:// doi.org/10.1029/2009JD012368.

Kleist, D. T., and K. Ide, 2015: An OSSE-based evaluation of hybrid variational-ensemble data assimilation for the NCEP GFS. Part II: 4DEnVar and hybrid variants. Mon. Wea. Rev., 143, 452-470, https://doi.org/10.1175/MWR-D-13-00350.1.

Kobayashi, S., and Coauthors, 2015: The JRA-55 Reanalysis: General specifications and basic characteristics. J. Meteor. Soc. Japan, 93, 5-48, https://doi.org/10.2151/jmsj.2015-001.

Kraichnan, R. H., 1967: Inertial ranges in two-dimensional turbulence. Phys. Fluids, 10, 1417-1423, https://doi.org/10.1063/ 1.1762301 .

Lauritzen, P. H., A. A. Mirin, J. Truesdale, K. Raeder, J. L. Anderson, J. Bacmeister, and R. B. Neale, 2011: Implementation of new diffusion/filtering operators in the CAM-FV dynamical core. Int. J. High Perform. Comput. Appl., 26, 63-73, https://doi.org/ 10.1177/1094342011410088.

Lei, L., and J. S. Whitaker, 2016: A four-dimensional incremental analysis update for the ensemble Kalman filter. Mon. Wea. Rev., 144, 2605-2621, https://doi.org/10.1175/ MWR-D-15-0246.1.

Leutbecher, M., and Coauthors, 2017: Stochastic representations of model uncertainties at ECMWF: State of the art and future vision. Quart. J. Roy. Meteor. Soc., 143, 2315-2339, https:// doi.org/10.1002/qj.3094.

Li, Q., and E. Lindborg, 2018: Weakly and strongly nonlinear mesoscale dynamics close to the tropopause? J. Atmos. Sci., 75, 1215-1229, https://doi.org/10.1175/JAS-D-17-0063.1.

Lilly, D. K., 1972: Numerical simulation studies of two-dimensional turbulence. II. Stability and predictability studies. Geophys. Fluid Dyn., 4, 1-28, https://doi.org/10.1080/03091927208236087.

- 1983: Stratified turbulence and the mesoscale variability of the atmosphere. J. Atmos. Sci., 40, 749-761, https://doi.org/ 10.1175/1520-0469(1983)040<0749:STATMV>2.0.CO;2.

Lindborg, E., 1999: Can the atmospheric kinetic energy spectrum be explained by two-dimensional turbulence? J. Fluid Mech., 388, 259-288, https://doi.org/10.1017/S0022112099004851.

Long, C. S., M. Fujiwara, S. Davis, D. M. Mitchell, and C. J. Wright, 2017: Climatology and interannual variability of dynamic variables in multiple reanalyses evaluated by the SPARC Reanalysis Intercomparison Project (S-RIP). Atmos. Chem. Phys., 17, 14 593-14 629, https://doi.org/10.5194/acp-17-145932017.

Lorenz, E. N., 1969: The predictability of a flow which possesses many scales of motion. Tellus, 21, 289-307, https://doi.org/ 10.3402/tellusa.v21i3.10086. 
Lynch, P., and X.-Y. Huang, 1992: Initialization of the HIRLAM model using a digital filter. Mon. Wea. Rev., 120, 1019-1034, https://doi.org/10.1175/1520-0493(1992)120<1019:IOTHMU> 2.0.CO;2.

Nastrom, G. D., and K. S. Gage, 1985: A climatology of atmospheric wavenumber spectra of wind and temperature observed by commercial aircraft. J. Atmos. Sci., 42, 950-960, https://doi.org/10.1175/1520-0469(1985)042<0950:ACOAWS> 2.0.CO;2.

,-- , and W. H. Jasperson, 1984: Kinetic energy spectrum of large- and mesoscale atmospheric processes. Nature, 310, 3638, https://doi.org/10.1038/310036a0.

O'Gorman, P. A., and T. Schneider, 2007: Recovery of atmospheric flow statistics in a general circulation model without nonlinear eddy-eddy interactions. Geophys. Res. Lett., 34, L22801, https://doi.org/10.1029/2007GL031779.

Palmer, T. N., R. Buizza, F. Doblas-Reyes, T. Jung, M. Leutbecher, G. J. Shutts, M. Steinheimer, and A. Weisheimer, 2009: Stochastic parametrization and model uncertainty. ECMWF Tech. Memo. 598, 42 pp.

Petersen, R. A., 2016: On the impact and benefits of AMDAR observations in operational forecasting-Part I: A review of the impact of automated aircraft wind and temperature reports. Bull. Amer. Meteor. Soc., 97, 585-602, https://doi.org/ 10.1175/BAMS-D-14-00055.1.

Randall, D. A., 2017: An Introduction to Numerical Modeling of the Atmosphere. Randall, 504 pp., http://hogback.atmos.colostate.edu/ group/dave/at604pdf/AT604_LaTeX_Book.pdf.

Sanchez, C., K. D. Williams, G. Shutts, and M. Collins, 2014: Impact of a stochastic kinetic energy backscatter scheme across time-scales and resolutions. Quart. J. Roy. Meteor. Soc., 140, 2625-2637, https://doi.org/10.1002/qj.2328.

Sandu, I., and Coauthors, 2019: Impacts of orography on largescale atmospheric circulation. npj Climate Atmos. Sci., 2, 10, https://doi.org/10.1038/s41612-019-0065-9.

Shutts, G., 2005: A kinetic energy backscatter algorithm for use in ensemble prediction systems. Quart. J. Roy. Meteor. Soc., 131, 3079-3102, https://doi.org/10.1256/qj.04.106.

_ M. Leutbecher, A. Weisheimer, T. Stockdale, L. Isaksen, and M. Bonavita, 2011: Representing model uncertainty: Stochastic parameterizations at ECMWF. ECMWF Newsletter, No. 129, ECMWF, Reading, United Kingdom, 19-24.

Skamarock, W. C., 2004: Evaluating mesoscale NWP models using kinetic energy spectra. Mon. Wea. Rev., 132, 3019-3032, https://doi.org/10.1175/MWR2830.1.
_ S.-H. Park, J. B. Klemp, and C. Snyder, 2014: Atmospheric kinetic energy spectra from global high-resolution nonhydrostatic simulations. J. Atmos. Sci., 71, 4369-4381, https:// doi.org/10.1175/JAS-D-14-0114.1.

Slivinski, L. C., G. P. Compo, J. S. Whitaker, P. D. Sardeshmukh, J.-W. A. Wang, K. Friedman, and C. McColl, 2019: What is the impact of additional tropical observations on a modern data assimilation system? Mon. Wea. Rev., 147, 2433-2449, https:// doi.org/10.1175/MWR-D-18-0120.1.

Smith, K. S., 2004: Comments on "The $k^{-3}$ and $k^{-5 / 3}$ energy spectrum of atmospheric turbulence: Quasigeostrophic two-level model simulation.' J. Atmos. Sci., 61, 937-942, https://doi.org/ 10.1175/1520-0469(2004)061<0937:COTKAE > 2.0.CO;2.

Takacs, L. L., M. J. Suárez, and R. Todling, 2018: The stability of incremental analysis update. Mon. Wea. Rev., 146, 3259-3275, https://doi.org/10.1175/MWR-D-18-0117.1.

Terasaki, K., and H. Tanaka, 2009: Characteristics of the kinetic energy spectrum of NICAM model atmosphere. SOLA, $\mathbf{5}$, 180-183, https://doi.org/10.2151/sola.2009-046.

Thatcher, D. R., and C. Jablonowski, 2016: A moist aquaplanet variant of the Held-Suarez test for atmospheric model dynamical core. Geosci. Model Dev., 9, 1263-1292, https:// doi.org/10.5194/gmd-9-1263-2016.

Tompkins, A. M., and J. Berner, 2008: A stochastic convective approach to account for model uncertainty due to unresolved humidity variability. J. Geophys. Res., 113, D18101, https:// doi.org/10.1029/2007JD009284.

Tung, K. K., 2004: Reply. J. Atmos. Sci., 61, 943-948, https:// doi.org/10.1175/1520-0469(2004)061<0943:R>2.0.CO;2. , and W. W. Orlando, 2003: The $k^{-3}$ and $k^{-5 / 3}$ energy spectrum of atmospheric turbulence: Quasigeostrophic two-level model simulation. J. Atmos. Sci., 60, 824-835, https://doi.org/10.1175/ 1520-0469(2003)060<0824:TKAKES $>2.0 . C O ; 2$.

Wang, J.-W. A., P. D. Sardeshmukh, G. P. Compo, J. S. Whitaker, L. C. Slivinski, C. M. McColl, and P. J. Pegion, 2019: Sensitivities of the NCEP Global Forecast System. Mon. Wea. Rev., 147, 1237-1256, https://doi.org/10.1175/MWR-D-18-0239.1.

Zhang, F., C. Snyder, and R. Rotunno, 2003: Effects of moist convection on mesoscale predictability. J. Atmos. Sci., 60, 1173-1185, https://doi.org/10.1175/1520-0469(2003)060<1173: EOMCOM $>2.0 . \mathrm{CO} ; 2$.

— N. Bei, R. Rotunno, C. Snyder, and C. C. Epifanio, 2007: Mesoscale predictability of moist baroclinic waves: Convectionpermitting experiments and multistage error growth dynamics. J. Atmos. Sci., 64, 3579-3594, https://doi.org/10.1175/JAS4028.1. 